\title{
Eletrofiação de Polímeros em Solução. Parte II: Aplicações e Perspectivas
}

\author{
Rodrigo G. F. Costa \\ Departamento de Química, UFSCar
}

Juliano E. de Oliveira, Gustavo F. de Paula

Departamento de Engenharia de Materiais, UFSCar

\author{
Paulo H. de S. Picciani \\ Instituto de Macromoléculas, UFRJ
}

\section{Eliton S. de Medeiros}

Departamento de Engenharia de Materiais, UFPB

\author{
Cauê Ribeiro, Luiz H. C. Mattoso \\ Embrapa Instrumentação, São Carlos
}

\begin{abstract}
Resumo: Em artigo de revisão anterior ${ }^{[1]}$, o processo de eletrofiação foi discutido, incluindo suas bases teóricas e experimentais, e a obtenção de diferentes nanofibras de materiais poliméricos. Neste segundo artigo de revisão, são abordados os aspectos relacionados à aplicação de materiais eletrofiados em diferentes áreas, como médica, agrícola, sensores, processamento de outros materiais, entre outras. São também discutidas as técnicas de caracterização utilizadas mais frequentemente nestes materiais, e suas potencialidades. Esta segunda revisão é complementar à anterior e segue, em seus aspectos gerais, a mesma terminologia.
\end{abstract}

Palavras-chave: Eletrofiação, nanocompósitos, catálise, sensores, blendas, microscopia, espalhamento de raio X.

\section{Electrospinning of Polymers in Solution. Part II: Applications and Perspectives}

Abstract: In our previous review ${ }^{[1]}$, the process of electrospinning was discussed on the basis of theoretical and experimental aspects toward preparation of different polymeric nanofibers. In this second review, we focus on the aspects related to the application of electrospun materials in various fields such as medicine, agriculture, sensors, and processing of other materials. We discuss the most often characterization techniques used for these materials and their potential. This review complements the previous one and uses the same terminology.

Keywords: Electrospinning, nanocomposites, catalysis, sensors, blends, microscopy, X ray scattering.

\section{Aplicações}

Aplicações da eletrofiação no processamento de outros materiais a partir de precursores poliméricos

A enorme gama de possibilidades abertas pelo processo de eletrofiação permite que diferentes aplicações sejam derivadas destes materiais, desde aplicações objetivas para o polímero em questão até outras, não tão usuais, como o processamento de precursores de outros materiais.

Assim, uma das aplicações do processo de eletrofiação é a preparação de nanofibras cerâmicas através do tratamento térmico dos compósitos eletrofiados. Neste caso, a solução precursora contém obrigatoriamente um polímero, o material cerâmico e o solvente ${ }^{[2-5]}$. Com este objetivo, $\mathrm{Li}$ e $\mathrm{Xia}^{[3]}$ prepararam nanofibras de $\mathrm{TiO}_{2}$ anatase utilizando uma solução precursora contendo poli(vinilpirrolidona) (PVP), tetraisopropóxido de titânio e etanol. As mantas obtidas foram calcinadas em temperatura de $500{ }^{\circ} \mathrm{C}$ resultando em nanofibras de $\mathrm{TiO}_{2}$ anatase com diâmetros na faixa de 20 a $200 \mathrm{~nm}$. Azad ${ }^{[5]}$ fabricou nanofibras transparentes de alumina $\left(\mathrm{Al}_{2} \mathrm{O}_{3}\right)$ utilizando uma solução precursora de 2,4 - pentanodionato de alumínio em acetona misturada com uma solução de PVP solubilizado em etanol. Estes compósitos foram tratados termicamente, aquecidos e sinterizados, nas temperaturas de 1000,1300 e $1500{ }^{\circ} \mathrm{C}$ por 1 hora em dois estágios. Assim, os autores conseguiram preservar a morfologia do compósito eletrofiado, obtendo nanofibras de alumina cristalinas e com alta pureza $^{[5]}$.

Assim como as nanofibras cerâmicas, as nanofibras e os nanofios metálicos têm propriedades únicas ${ }^{[-8]}$, as quais fazem com que estes materiais tenham potenciais aplicações nas mais diversas áreas como nanoeletrônica, nanofiltração e nanossensores ${ }^{[9-11]}$ Com isso alguns autores estão se dedicando ao estudo de fibras metálicas obtidas através do método de eletrofiação ${ }^{[9-13]}$.

Bognitzki et al. ${ }^{[9]}$ reportaram a obtenção de nanofibras de cobre obtidas através da eletrofiação de uma solução contendo poli(vinil butiral), $\mathrm{PVB}$, e nitrato de cobre, $\mathrm{Cu}\left(\mathrm{NO}_{3}\right)_{2}$. O processo de eletrofiação foi empregado sob condições padrão obtendo-se fibras com um diâmetro médio de $550 \mathrm{~nm}$. Após tratamento térmico em ar atmosférico os autores notaram a degradação do PVB. Um tratamento térmico posterior, em atmosfera de hidrogênio, resultou na formação de nanofibras de cobre com diâmetro da ordem de

Autor para correspondência: Rodrigo G. F. Costa, Departamento de Química, Universidade Federal de São Carlos - UFSCar,

Rod. Washington Luís, Km 235, CEP 13565-905, São Carlos, SP, Brasil, e-mail: guerreiro_rodrigo@yahoo.com.br 
$270 \mathrm{~nm}$. Essa redução no diâmetro foi atribuída à perda de massa de PVB durante a etapa de sua degradação ${ }^{[9]}$.

Pol et al. ${ }^{[10]}$ obtiveram nanofibras de ouro a partir da calcinação de mantas de poli(ácido acrílico), PAA, contendo $\mathrm{HAuCl}_{4} \cdot 3 \mathrm{H}_{2} \mathrm{O}$ como precursor. As fibras apresentaram diâmetro médio da ordem de $50 \mathrm{~nm}$ a poucos micrometros. As propriedades elétricas e eletroquímicas do ouro foram confirmadas por medidas de condutividade elétrica e voltametria cíclica.

Wang e Liu em 2009 ${ }^{[1]}$ investigaram nanofibras de $\mathrm{SnO}_{2}$ dopadas com ferro obtidas por eletrofiação como materiais sensores a etanol. Inicialmente os autores prepararam uma solução de cloreto de estanho, cloreto de ferro e PVP. Esta solução foi eletrofiada e calcinada para remoção da fase orgânica. As nanofibras com diâmetro médio da ordem de $90 \mathrm{~nm}$ foram empregadas num sistema de sensoriamento de gases para detecção de etanol. Os sensores apresentaram limite de detecção da ordem de 10 ppm de etanol.

Wu e seu grupo ${ }^{[13]}$ estudaram as propriedades magnéticas de nanofibras de ferro, níquel e cobalto obtidas por eletrofiação. As nanofibras metálicas apresentaram propriedades ferromagnéticas com aumento em sua coercividade. Os autores também obtiveram fibras metálicas alinhadas e uma fibra da liga ternária FeNiCo. Estas novas nanofibras magnéticas têm potencial para emprego em sensores magnéticos, magnetos flexíveis e dispositivos de armazenamento de dados.

\section{Aplicações da eletrofiação no processamento de nanocompósitos de matriz polimérica e blendas}

O processo de eletrofiação também tem sido usado para preparar nanocompósitos com diferentes aplicações ${ }^{[14-16]}$. Dentre os sistemas matriz polimérica/carga pesquisados, vários trabalhos enfocam a utilização de nanotubos de carbono como reforços na matriz polimérica devido às ótimas propriedades destes materiais, como por exemplo, mecânica e elétrica ${ }^{[16-17]}$. Entretanto, as propriedades desses nanocompósitos estão diretamente relacionadas ao alinhamento do nanotubo na matriz polimérica, o qual é difícil de ser obtido por meio de métodos convencionais. Com este objetivo Ko et al. ${ }^{[16]}$ obtiveram nanocompósitos com os nanotubos de carbono paralelos ao eixo das fibras de poli(acrilonitrila), PAN. Sen et al. ${ }^{[17]}$ prepararam nanofibras na faixa de 50-100 $\mathrm{nm}$ de poliestireno (PS) reforçadas com nanotubos de carbono e obtiveram nanotubos de carbono paralelos ao eixo das fibras de poliestireno/nanotubo. Com a funcionalização do éster do nanotubo para depois ser eletrofiado, obtiveram uma nanofibra de poliuretana (PU)/nanotubo com um aumento da resistência à tração e do módulo elástico de 104 e 250\%, respectivamente, em relação à membrana eletrofiada de $\mathrm{PU}$ pura.

Shao et al. ${ }^{[14]}$ prepararam nanofibras de $\mathrm{PVA} / \mathrm{SiO}_{2} \mathrm{com}$ diâmetros na faixa de 200-400 nm. Os autores observaram que com o aumento do carregamento de sílica as nanofibras apresentaram uma maior estabilidade térmica e uma diminuição da cristalinidade. Son et al. ${ }^{[18]}$ reportaram nanofibras de acetato de celulose/Ag com propriedades bactericidas. Neste caso, as nanopartículas de Ag com diâmetros médios de $21 \mathrm{~nm}$ foram geradas através da irradiação das mantas com lâmpada UV. Estas mantas apresentaram uma atividade antibacteriana de 99,9\% depois de 18 horas de incubação. Costa et al. ${ }^{[19]}$ demonstraram que é possível preparar nanocompósitos de PVA/Ag utilizando o processo de eletrofiação, sem o uso de agentes redutores ou qualquer tratamento térmico posterior para reduzir a prata. As nanofibras obtidas, com diâmetros na faixa de 50-160 nm, apresentaram atividade antibacteriana frente às bactérias Escherichia coli e Staphylococcus aureus.

Em 2008, Medeiros et al. ${ }^{[15]}$ verificaram que as propriedades mecânicas (módulo de elasticidade e resistência à tração) dos nanocompósitos de PVA reforçados com nanofibrilas de celulose
(CnF) foram aumentadas em cerca de $240 \%$ através da adição de $7 \%$ em peso de $\mathrm{CnF}$ sem mudanças significativas nos valores de elongação na ruptura.

$\mathrm{Li}$ et al. ${ }^{[20]}$ estudaram nanofibras de poliamida 6 (PA6) e nanocompósitos de PA6/argila organicamente modificada (MMT) (Cloisite-30B). Os autores obtiveram nanofibras de compósitos com diâmetros de aproximadamente $100 \mathrm{~nm}$ e observaram que para estas fibras, o módulo de elasticidade aumentou $70 \%$ quando comparado com as fibras de PA6. Lee et al. demonstraram que a incorporação de MMT na matriz de PVA melhorou a estabilidade térmica e a resistência à tração do polímero ${ }^{[21]}$.

De forma semelhante à pesquisa em nanocompósitos, a pesquisa de blendas poliméricas visa o desenvolvimento de novos materiais com propriedades intermediárias ou mesmo superiores àquelas observadas nos constituintes puros ${ }^{[22-25]}$. Além disso, nanoestruturas poliméricas apresentam novas propriedades de interesse em diversas áreas como liberação controlada, biomateriais, estrutural, sensores e de embalagens ${ }^{[26]}$. Com isso, diversos grupos de pesquisa estudam nanofibras de blendas poliméricas obtidas por eletrofiação ${ }^{[26,27]}$. Estes estudos pretendem aliar as propriedades das blendas poliméricas ao comportamento dos materiais na escala nanométrica.

Blendas poliméricas podem ser classificadas em miscíveis ou imiscíveis. Ao se trabalhar com uma blenda imiscível durante o processo de eletrofiação, podemos obter uma morfologia de fase dispersa em matriz e outra de fase co-contínua. A eletrofiação, partindo de homopolímeros de baixa massa molar tende à formação de uma estrutura de fase dispersa numa matriz devido à maior mobilidade das moléculas ${ }^{[26-30]}$.

Choi et al. em 2008 ${ }^{[29]}$ aliaram as propriedades mecânicas da blenda de poli( $\varepsilon$-caprolactona)/colágeno à excelente biocompatibilidade e propriedade de adesão celular do colágeno. Os autores verificaram que as nanofibras obtidas permitiram uma orientação durante o crescimento de células do tecido ósseo humano. Nanofibras da blenda de quitosana e poli(óxido de etileno) (PEO) foram reportadas por Desai et al. em 2009 ${ }^{[30]}$. As blendas desenvolvidas apresentaram excelentes propriedades em aplicações envolvendo a membranas de filtração para íons de cromo em solução. Chen et al. ${ }^{[28]}$ estudaram as propriedades das blendas de náfion e $\mathrm{PAA}^{[28]}$. A eletrofiação do polieletrólito náfion requer a formação de uma blenda com outro polímero. A adição de um segundo polímero à solução de náfion tem o objetivo inicial de promover um grau de emaranhamento de cadeia dos polímeros em solução necessário no processo de eletrofiação. No sistema estudado pelos autores, a adição de PAA à solução de náfion aumenta sua força iônica, suprime a formação de grandes agregados e, consequentemente, aumenta a viscosidade da solução da blenda. As nanofibras formadas com diâmetro médio de $90 \mathrm{~nm}$ apresentaram elevada estabilidade em água, o que indica uma variedade de aplicações como em células combustíveis.

\section{Aplicações da eletrofiação em engenharia tecidual médica}

O emprego de nanofibras de materiais poliméricos biocompatíveis na substituição de tecidos ou órgãos em seres humanos, objetiva um rápido processo de recuperação através da otimização na interação tecido-implante. Entre suas propriedades mais importantes para a utilização em tecidos, podemos citar a elevada área superficial, boa elasticidade e permeação a gases. $\mathrm{Na}$ literatura foi reportada a aplicação destas nanofibras como parte de próteses $^{[31]}$, vasos sanguíneos ${ }^{[32]}$, pele $\operatorname{artificial~}^{[33]}$, biossensores $^{[34]}$ e liberação controlada de fármacos ${ }^{[35]}$, scaffolds $^{[36]}$ e curativos ${ }^{[37]}$. Alguns autores já vêm apontando a incorporação de fatores de crescimento ${ }^{[38,39]}$, fármacos ${ }^{[40]}$, enzimas ${ }^{[39-42]}$ ou até mesmo fragmentos de $\mathrm{DNA}^{[43]}$ nas nanofibras controlando a forma 
como estes materiais interagem com as células e o organismo do individuo $^{[39]}$.

A pesquisa de materiais para substituição de pele é objeto de estudo em vários grupos de pesquisa ao redor do mundo ${ }^{[44-47]}$. Khil et al. ${ }^{[37]}$ pesquisaram a aplicação de nanofibras de poliuretana como pele artificial. Elas apresentaram controle da evaporação de água e excelente permeabilidade a oxigênio. Além disso, nenhuma toxicidade ou permeabilidade a agentes exógenos foi observada. Os estudos histológicos demonstraram uma maior taxa de crescimento epitelial. Em seus trabalhos Luu et al. ${ }^{[43]}$ obtiveram mantas eletrofiadas de um copolímero em bloco de poli(lactídeo)poli(etilenoglicol) (PLAG) contendo fatores de crescimento e diferenciação celular.

Embora infecções sejam complicações potenciais, a possível formação de uma cápsula fibrosa entre o material e o tecido também pode levar à rejeição e até mesmo expulsão do implante ${ }^{[39]}$. A incorporação de fármacos a matrizes poliméricas tem a principal finalidade de tratar estas infecções e processos inflamatórios a fim de se evitar a rejeição do implante. Kim et al. ${ }^{[48]}$ incorporaram um antibiótico (Mefoxin) a solução de PLAG em DMF e notaram que este influência na morfologia e diâmetro das fibras. A adição de 5\% em peso de antibiótico levou ao aparecimento de contas e a uma redução no diâmetro médio das fibras. Através de estudos por UVVIS e 1H-RMN os autores reportaram que a estrutura do fármaco se manteve inalterada mesmo após a eletrofiação. Testes in vitro indicaram que o fármaco também não perdeu sua funcionalidade mesmo após o processo de eletrofiação.

Umatendênciacada vezmais frequentenapesquisa emengenharia de tecidos é o desenvolvimento de scaffolds tridimensionais em nanoescala ${ }^{[36]}$. Estas matrizes têm como principal objetivo o controle do crescimento celular. A estrutura das nanofibras obtida através da técnica de eletrofiação é ideal, pois sua dimensão se assemelha a dos componentes da matriz extracelular, e pode simular um ambiente fibrilar. Isso permite uma elevada organização celular, redução da apoptose e necrose celular e permanência da funcionalidade das células ${ }^{[39]}$. As nanofibras apresentam elevada porosidade e relação área/volume, o que possibilitam uma boa adesão e proliferação celular neste ambiente ${ }^{[49]}$.

Baseando-se nestes conceitos, $\mathrm{Xu}$ et al. ${ }^{[50]}$ realizaram estudos sobre crescimento e proliferação de células da artéria coronária humana em matrizes constituídas de filmes e nanofibras obtidas por eletrofiação do copolímero poli(L-lactídeo-co- $\varepsilon$-caprolactona) (75:25). As fibras apresentaram diâmetro médio da ordem de $500 \mathrm{~nm}$ e topografia alinhada. Esta arquitetura foi desenvolvida com o intuito de imitar o ambiente extracelular presente na camada intermediária da artéria humana. Com isso os autores investigaram a interação entre as células do músculo liso da artéria coronária com filmes obtidos por casting como controle e as nanofibras. Os resultados obtidos indicam que as células se orientaram no mesmo sentido de orientação das nanofibras, além de que a adesão e taxa de proliferação das mesmas foi significativamente superior no caso das nanofibras quando comparado com o controle.

Estes resultados sugerem um efeito sinérgico das propriedades do copolímero sintético biodegradável com as dimensões e arquitetura em nanoescala que imitam o ambiente extracelular natural e desta forma replica as condições encontradas pelas células na estrutura cardiovascular. Uma análise da literatura ${ }^{[31,42,51,52]}$ torna evidente que as células respondem de forma diferenciada quando sujeitas a matrizes de crescimento tridimensional quando comparadas com as matrizes bidimensionais. Esta diferença é atribuída muitas vezes ao mimetismo do ambiente extracelular natural encontrado pelas células quando submetidas às nanofibras. Um próximo passo neste campo de pesquisas considera a melhoria das propriedades mecânicas das nanofibras através da incorporação de cargas $^{[32,36]}$ ou mesmo pós-tratamento térmico destas nanoestruturas ${ }^{[53,54]}$.

\section{Aplicações da eletrofiação em sensores}

No que tange as aplicações em sensores, o aumento da área específica é geralmente entendido como um meio para aumentar a sensibilidade dos dispositivos. Além disso, a possibilidade de controlar o alinhamento das fibras também é vista como uma maneira de controlar a resposta sensorial.

O primeiro relato de uso de nanofibras poliméricas para aplicação em sensores é devido a Wang et al., em 2002 ${ }^{[55]}$. Um sensor fluorimétrico à base de PAA com grupo pireno pendente foi utilizado para detecção de $\mathrm{Hg}^{2+}, \mathrm{Fe}^{3+}$, e 2,4-dinitrotolueno com duas ou três ordens de magnitude em melhoria da sensibilidade em relação a filmes preparados anteriormente. Em 2004, Liu et al.

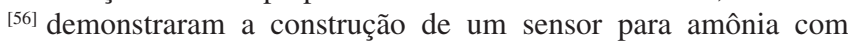
sensibilidade melhorada a partir da manipulação de uma única fibra de poli(anilina) (PANI)/poli(óxido de etileno) (PEO), preparada por eletrofiação. Wang apresentou mais uma inovação na área, com o relato de um sensor fluorimétrico baseado em uma manta de acetato de celulose eletrofiada recoberta com um filme automontado de (PAH/H-PURET) ${ }^{[57]}$. A sensibilidade neste caso foi menor que o valor anteriormente obtido, porém demonstra a viabilidade da automontagem sobre nanofibras para obtenção de dispositivos.

Luoh e $\mathrm{Hahn}^{[58]}$ utilizaram nanofibras com partículas de óxido encapsuladas para melhorar a adsorção de $\mathrm{CO}_{2}$ e permitir detecção melhorada por FTIR. Manesh et al. ${ }^{[59]}$ fabricaram nanofibras de poli(difenil anilina) compostas com poli(metil metacrilato) para uso como sensor resistivo de amônia; o resultado obtido é comparável ao de sensores baseados em filmes de polímeros condutores. Tao et al. ${ }^{[60]}$ utilizaram uma técnica mista de sol-gel seguido de eletrofiação para produzir nanofibras de porfirinas ligadas por organossilanos. Tais fibras foram utilizadas para detecção fluorimétrica de trinitrotolueno em fase vapor, com desempenho comparável ao obtido por sensores baseados em filmes de polímeros condutores. Haynes e Gouma ${ }^{[61]}$ preparam sensores à base de polianilina na forma leucoesmeraldina embebida em acetato de celulose e aplicaram à detecção de $\mathrm{NO}_{2} \mathrm{e}$ $\mathrm{pH}$ da fase vapor de soluções aquosas, visando simular a análise destes parâmetros na respiração humana. Os sensores tiveram um tempo de resposta típico de 5 minutos, porém tornaram-se rapidamente envenenados por $\mathrm{NO}_{2}$.

Yoshimatsu et al. ${ }^{[62]}$ prepararam nanofibras de poliestireno dopado com 9,10-difenilantraceno para operar como matriz para sensor cintilométrico de $(S)$-propanolol com base em reconhecimento molecular por partículas molecularmente impressas. Foi demonstrada a viabilidade da técnica, considerada pelos autores como um método efetivo para preparação de sensores à base de nanofibras operando por reconhecimento molecular. Kuo et al. ${ }^{[63]}$ avaliaram nanofibras alinhadas de poli(metil metacrilato) contendo polifluorenos para detecção fluorimétrica de DNA; os autores declaram que o sensor baseado em nanofibras apresenta sensibilidade muito maior que o baseado em filme fabricado por spin-coating do mesmo material, porém os dados apresentados ilustram uma sensibilidade apenas ligeiramente maior.

Tiwari et al. ${ }^{[64]}$ demonstraram a fabricação de sensores de deformação baseados em nanofibras de poli( $\varepsilon$-caprolactona) (PCL) incorporando negro-de-fumo condutivo. Conforme as fibras são alongadas axialmente, ocorre uma redução da percolação das partículas de negro-de-fumo, reduzindo a condutividade do material. Os autores apresentam uma modelagem do processo considerando a aplicação de uma membrana deste material como filtro, sendo o entupimento do filtro (e consequente estiramento da membrana) monitorado pela variação da condutividade. Rathfon et al. ${ }^{[65]}$ 
desenvolveram sensores fluorimétricos para detecção do gás cloroSarin baseados em filmes e nanofibras de poliestireno incorporando pirenoimina. Demonstrou-se que a sensibilidade na detecção baseada em filmes e fibras é oito vezes maior do que em solução, mas não houve diferença entre a morfologia fibrilar ou em filmes.

Yoon et al. ${ }^{[6]}$ apresentam uma estratégia de diferenciação colorimétrica de solventes orgânicos baseada na fabricação combinatória de mantas eletrofiadas de PEO contendo concentrações variadas de dois derivados de polidiacetileno. Diferentes padrões de cores são obtidos conforme o solvente ao qual as mantas foram expostas. Recentemente, Picciani et al. ${ }^{[67]}$ produziram nanofibras condutoras de PANI/PLA com diâmetros na faixa entre 100 e $1000 \mathrm{~nm}$. Estas nanofibras foram preparadas a partir das soluções das blendas de PANI dopada com TSA e PLA em hexafluorisopropanol. As nanofibras obtidas são homogêneas, indicando que existe uma boa interação entre a PANI e o PLA na blenda. Apesar de a condutividade elétrica ser menor nas nanofibras do que nos respectivos filmes preparados por casting, estes materiais são bastante promissores para serem usados em sensores, uma vez que a condutividade dos materiais é fortemente dependente do diâmetro das fibras. Costa et al. ${ }^{[68]}$ produziram filmes de poli(fluoreto de vinilideno), PVDF, na fase ferroelétrica $\beta$, por eletrofiação. Os autores verificaram que os filmes obtidos por eletrofiação são compostos por nanofibras na fase $\beta$ e com os eixos das cadeias poliméricas orientados preferencialmente ao longo das fibras. Devido à presença exclusiva da fase $\beta$, estas nanofibras têm potencial para serem usadas em atuadores e sensores.

Em resumo, diversos princípios de transdução (fluorimétrico, condutométrico, colorimétrico) foram avaliados para diversos analitos, principalmente gases e solventes. Nos casos em que a comparação foi feita com filmes do mesmo material, não se observou vantagens claras no uso de nanofibras, com raras exceções. Com o constante crescimento dos estudos de aplicação de nanofibras para aplicação em sensores, em breve será possível esclarecer em quais situações a morfologia fibrilar efetivamente apresenta vantagem técnica.

\section{Aplicações da eletrofiação em catálise}

Aplicação de nanofibras em catálise é uma idéia que naturalmente surge quando se considera a alta razão comprimentodiâmetro destas estruturas. Porém polímeros normalmente não possuem propriedades que impliquem em efeitos catalíticos. Deste modo, busca-se então o carregamento das fibras com catalisadores típicos, tais como partículas metálicas, de semicondutores, e enzimas; as nanofibras neste caso oferecem um suporte de alta área específica e razoável estabilidade, aspectos fundamentais para sistemas catalíticos.

Em 2004, Demir et al. ${ }^{[69]}$ prepararam mantas de poli(acrilonitrilaco-ácido acrílico) recobertas de nanopartículas de paládio, estas preparadas pela redução (posterior à eletrofiação) de $\mathrm{PdCl}_{2}$ com hidrazina ${ }^{[69]}$. Paládio é um catalisador seletivo para hidrogenação de dienos e olefinas, dentre outras aplicações. O controle do teor de nanopartículas de paládio pode ser feito tanto pela regulação do teor de $\mathrm{PdCl}_{2}$ adicionado quanto pelo teor de ácido acrílico no polímero eletrofiado. Chen et al. ${ }^{[70]}$ avaliaram a formação de nanopartículas de paládio e prata em mantas de poli(acrilonitrila) através de irradiação com microondas. Controlando a composição da solução precursora, o diâmetro das nanopartículas pôde ser controlado. Neste trabalho porém, não foi avaliada a atividade catalítica das mantas.

Zhou et al. ${ }^{[71]}$ avaliaram a atividade fotocatalítica de nanopartículas de $\mathrm{ZnS}$ sintetizadas sobre mantas de PAN e poli(metil metacrilato-co-butil metacrilato-co-ácido acrílico) obtidas de soluções em dimetilformamida. As mantas contendo ZnS apresentaram atividade na foto-oxidação de fenol em meio aquoso mesmo após seis ciclos de uso. Em 2010, Costa et al. ${ }^{[72]}$ estudaram as propriedades dos nanocompósitos de $\mathrm{PVA} / \mathrm{TiO}_{2}$ anatase. Os autores observaram que quanto maior o carregamento do PVA com $\mathrm{TiO}_{2}$, maior o número de contas nas fibras. Entretanto, verificaram que a presença de pequenas porcentagens de $\mathrm{TiO}_{2}$ nas fibras mudou a absorção da luz UV e diminuiu significativamente o tempo de fotodegradação do polímero.

Catálise enzimática foi avaliada por $\mathrm{Wu}$ et al. ${ }^{[73]}$ através da eletrofiação de uma solução de celulase com PVA em ácido acético diluído, seguido de reticulação com glutaraldeído. O material depositado em nanofibras apresentou rendimento catalítico superior à enzima livre e a filmes obtidos por evaporação do solvente na hidrólise de celulose em meio ácido. O catalisador pôde ser reutilizado, porém a atividade catalítica caiu a aproximadamente metade após seis ciclos de uso. Kim et al. ${ }^{[74]}$ desenvolveram um método de formação de agregados de enzima sobre nanofibras de uma blenda de poliestireno com poli(estireno-co-anidrido malêico), no qual os agregados enzimáticos de $\alpha$-quimotripsina reticulados com glutaraldeído, imobilizados nas nanofibras, apresentam maior estabilidade e maior atividade catalítica (nove vezes em relação a uma única camada de enzima) dado pelo aumento da quantidade de enzima imobilizada.

\section{Aplicações da eletrofiação em agricultura e purificação de águas}

Buscando o desenvolvimento de novos produtos com maior valor agregado voltados para o agronegócio, alguns grupos de pesquisa vêm publicando trabalhos relativos ao emprego de nanofibras obtidas pela técnica de eletrofiação em barreiras protetoras a pesticidas ${ }^{[75]}$, membranas de clarificação de suco de maçã $^{[76]}$, nanofibras de seda ${ }^{[77,78]}$ embalagens ativas ${ }^{[7,80]}$ e liberação controlada de vitaminas ${ }^{[80]}$.

Lee e Obendorf ${ }^{[75]}$ avaliaram o potencial de nanofibras de poliuretana como barreira a passagem de pesticidas, água e temperatura. Em seu trabalho eles observaram que a adição das nanofibras de poliuretana sob a superfície de tecidos convencionais empregados na manufatura de trajes de segurança para trabalhadores rurais trouxe uma melhora significativa nas propriedades de barreira do material.

Membranas constituídas de nanofibras de PET foram estudadas por Veleirinho e Silva ${ }^{[76]}$. Devido à sua elevada porosidade, relação área/volume e estrutura interconectada, estas membranas vêm sendo investigadas em processos de ultrafiltração de suco de maçã. Este processo tem como objetivo reduzir os custos da etapa de clarificação, além de dotar o produto final de propriedades organolépticas mais aceitáveis pelo consumidor.

Atualmente alguns grupos de pesquisa vêm avaliando o emprego de nanofibras de alguns polímeros em embalagens ativas. Por exemplo, Torres e colabores ${ }^{[78]}$ em seus trabalhos buscam obter nanofibras de zeína do milho, além da incorporação de antioxidantes como $\beta$-caroteno à matriz de zeína.

Além de antioxidantes alguns grupos buscam a incorporação de vitaminas a matrizes poliméricas. Taepaiboon et al. ${ }^{[80]}$ estudaram a adição e liberação das vitaminas A e E em nanofibras de acetato de celulose com diâmetro médio de $250 \mathrm{~nm}$.

\section{Técnicas de caracterização morfológica e estrutural}

\section{Microscopia}

A morfologia e a geometria (distribuição de diâmetros, orientação e alinhamento) das nanofibras eletrofiadas são caracterizadas utilizando-se basicamente técnicas microscópicas: 
microscopia eletrônica de varredura (MEV), microscopia eletrônica de emissão de campo (MEV-EC), microscopia eletrônica de transmissão (MET) e Microscopia de Força Atômica (MFA) ${ }^{[81-83] .}$ Dentre essas três técnicas, a microscopia eletrônica de varredura é a mais usada para verificar a morfologia e determinar a distribuição de diâmetros das fibras, por fornecer uma visão mais geral da amostra. As nanofibras com maiores diâmetros, de 200 a $1000 \mathrm{~nm}$, são observadas com magnificações de aproximadamente 15.000 vezes, enquanto que para as nanofibras com diâmetros menores que $200 \mathrm{~nm}$, são necessárias magnificações de pelo menos 20.000 vezes. Magnificações destas ordens não podem ser obtidas em alguns microscópios eletrônicos de varredura convencionais. Neste caso, faz-se necessário o uso de microscópios eletrônicos de varredura mais potentes ou com o uso de microscópios eletrônicos de emissão de campo. Além do que, para o caso de nanofibras poliméricas de fontes vegetais, tais como, amido, quitosana, e celulose ${ }^{[82]}$, aumentos desta ordem em microscópios de varredura convencionais podem degradar estes materiais. A microscopia eletrônica de transmissão é mais usada quando se deseja obter aumentos da ordem de 100.000 a 200.000 vezes, como por exemplo, em nanofibras políméricas com diâmetros de 10 a $50 \mathrm{~nm}$, e em nanocompósitos para se observar a distribuição das nanopartículas dispersas nas fibras ${ }^{[84,85]}$.

Jose et al. ${ }^{[85]}$ estudaram a morfologia das nanofibras de PA6/ nanotubos de carbono multiparedados por MEV e MET. Através das micrografias de MEV, observaram que com o aumento da concentração de nanotubos no nanocompósito o diâmetro da fibra diminuiu e fibras com diâmetros uniformes foram obtidas. As micrografias obtidas por MET mostraram que os nanotubos estão altamente alinhados.

A microscopia de força atômica (MFA) é mais empregada para se investigar a morfologia, a topologia e diversas propriedades locais em superfícies de materiais que necessitam de elevada resolução para a análise ${ }^{[86-89]}$.

Em 2004, Lee e Belcher ${ }^{[88]}$ reportaram a eletrofiação de um sistema constituído do bacteriófago anti-estreptavidina M13 e PVP. Constataram que após o processo de eletrofiação a estrutura do vírus nas fibras se manteve intacta. Segundo os autores estas fibras são ferramentas poderosas em aplicações que envolvam alta atividade biocatalítica como biomedicina e biossensores. Através de informações obtidas por MFA os autores constataram uma orientação paralela dos vírus ao longo do eixo longitudinal das fibras.

Em 2008, Wu et al. ${ }^{[89]}$ descreveram uma nova técnica de eletrofiação baseada num microscópio de força atômica assistido por alta voltagem. Através da aplicação de um alto potencial elétrico entre a ponta da agulha modificada pela solução polimérica e um substrato condutor os autores obtiveram nanofibras de PEO. Através deste método foi possível o controle espacial de deposição das fibras sobre um substrato. A Figura 1 ilustra de forma esquemática este processo de nanoeletrofiação.

\section{Espalhamento de raio $X$}

As técnicas de espalhamento de raio X são tidas como técnicas muito poderosas para a caracterização estrutural de materiais poliméricos. Dependendo do tamanho das estruturas analisadas e das informações a serem obtidas os espalhamento de raio $\mathrm{X}$ a altos ângulos (WAXS) ou o espalhamento de raio $\mathrm{X}$ a baixos ângulos (SAXS) podem ser utilizados, sendo que para fibras poliméricas eletrofiadas as duas técnicas podem ser utilizadas de modo complementar. As técnicas de espalhamento em geral permitem a obtenção de informações provenientes de uma maior área, ou quantidade, da amostra, sendo, desta forma, mais representativa e complementar às técnicas de microscopia abordadas anteriormente.

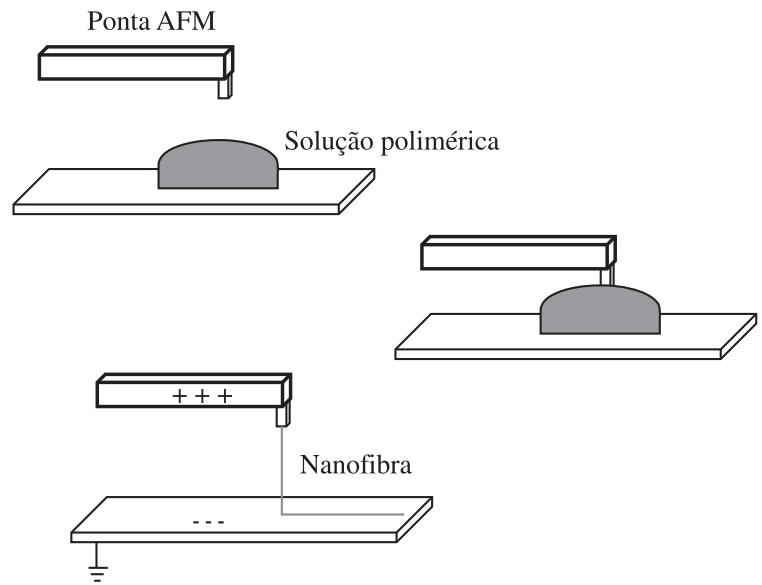

Figura 1. Representação esquemática do processo de nanoeletrofiação baseado em microscópio de força atômica.

A estrutura e cristalinidade de nanofibras de PLA e PA6 com diâmetro médio de $5 \mathrm{~nm}$ obtidas por eletrofiação foram estudadas por Dersch et al. ${ }^{[90]}$. Os autores observaram que as nanofibras apresentavam cristalinidade semelhante à encontrada na extrusão. Devido à rápida cristalização (na escala de microssegundos) causada por uma elevada taxa de evaporação do solvente mesmo as altas taxas de deformação elongacional características do processo de eletrofiação ${ }^{[2,9]}$ não possibilitam que os cristais formados sejam orientados nas nanofibras. Em seus trabalhos os autores mostraram que a orientação é inexistente nas nanofibras de PLA e fraca nas nanofibras de PA6, indicando assim que o processo de eletrofiação não favorece o aumento da cristalinidade do polímero.

No entanto, tratamentos posteriores nas mantas eletrofiadas podem aumentar a cristalinidade das fibras. Zong et al. ${ }^{[53]}$ estudaram $\mathrm{o}$ efeito de tratamentos termomecânicos na cristalinidade e propriedades mecânicas de nanofibras de PLA. Eles notaram que as fibras apresentavam baixa cristalinidade imediatamente após serem eletrofiadas. No entanto, através de um estiramento e aquecimento a altas temperaturas, foi possível a obtenção de nanofibras com maior grau de cristalinidade. Segundo análises posteriores de WAXS e SAXS, os autores encontraram fibras com até $40 \%$ de cristalinidade. Outros grupos de pesquisa estudaram o efeito de tratamentos térmicos em nanofibras de $\mathrm{PAN}^{[91]}$. Os resultados indicam um aumento significativo no grau de cristalinidade das fibras após tratamento realizado a temperaturas da ordem de 65 a $95{ }^{\circ} \mathrm{C}$.

Recentemente Lim et al. ${ }^{[54]}$ estudaram a cristalinidade de nanofibras de PCL por raio X e calorimetria diferencial exploratória. Além disso, eles empregaram a técnica MFA para se avaliar a morfologia da fase cristalina. Os autores ainda encontraram que fibras de menor diâmetro apresentam maior cristalinidade e grau de orientação molecular. O diâmetro das nanofibras, assim como sua cristalinidade, é influenciado pelo momento em que ocorre a cristalização das cadeias. Foi observado que caso as cadeias se cristalizem antes de alcançar o coletor metálico tem-se a formação de uma estrutura lamelar contendo fibrilas. Já no caso da cristalização ocorrer após as fibras alcançarem o coletor tem-se uma estrutura lamelar não orientada. Estes resultados contribuíram para se explicar as variações ocorridas nas propriedades mecânicas das nanofibras preparadas sob diferentes condições ${ }^{[54]}$.

Recentemente Picciani et al. ${ }^{[67]}$ compararam o grau de cristalinidade de nanofibras de diferentes composições de blendas de PLA e PANI obtidas por eletrofiação com os respectivos filmes obtidos por casting. Seus resultados corroboram com a interpretação 
de que as fibras apresentam significativamente menores valores de cristalinidade. Além disso, resultados posteriores de SAXS e MFA indicam que, além da menor cristalinidade, não houve separação de fase entre os componentes da fibra, como observado nos filmes ${ }^{[24]}$.

\section{Outras técnicas}

O termo análise térmica é frequentemente empregado para descrever as diversas técnicas de análise experimental que investigam o comportamento de uma amostra em função da temperatura. No campo de pesquisas relativo à eletrofiação de nanofibras poliméricas estas técnicas são rotineiramente empregadas para se avaliar o efeito do processamento nas propriedades do material como temperatura de transição vítrea e temperatura de inicio de degradação ${ }^{[53,92-94]}$.

Zong et al. ${ }^{[53]}$ compararam a temperatura de transição vítrea e cristalinidade de nanofibras de PLA obtidas por eletrofiação com amostras deste polímero sem qualquer tratamento através de análises de DSC. Os autores observaram uma redução na temperatura de transição vítrea que pode ser atribuída à elevada relação área/volume bem como ao efeito plastificante do ar durante a solidificação do polímero. O baixo grau de cristalinidade encontrado foi entendido como sendo o resultado de uma solidificação extremamente rápida das cadeias poliméricas submetidas a altas taxas de estiramento características do processo de eletrofiação.

A estrutura molecular e a identidade dos grupos funcionais presentes nas nanofibras podem ser elucidadas com a Espectroscopia de Infravermelho com Transformada de Fourier (FTIR). No caso de uma blenda ou de um nanocompósito, verifica-se a presença dos grupos funcionais dos dois materiais que constituem as nanofibras, assim como, pode-se observar a existência de interações intermoleculares entre os dois materiais ${ }^{[95]}$. Também, pode-se avaliar a influência do processo de eletrofiação na estrutura química dos materiais eletrofiados ${ }^{[95,96]}$.

\section{Sumário}

A aplicação de nanofibras poliméricas como precursoras de fibras cerâmicas ou metálicas é uma área com forte tendência à expansão, pois resultados satisfatórios vêm sendo obtidos e o potencial de aplicação é bastante expressivo. Muito pouco deste potencial já foi explorado, e espera-se que novidades surpreendentes surjam em breve, principalmente no campo da nanoeletrônica.

O desenvolvimento de nanocompósitos também guarda ainda muitas possibilidades de avanço, tanto em novos sistemas polímero/carga quanto na aplicação industrial destes materiais. Reforço de materiais de engenharia, membranas de filtração com alta seletividade, recobrimento de próteses médicas, nanoatuadores magnéticos, são apenas algumas das inúmeras possibilidades de aplicação destes compósitos nanoestruturados. Assim como com nanocompósitos, a busca de blendas nanoestruturadas visa criar materiais com combinação de propriedades não encontrada nos materiais de partida. Tanto materiais aprimorados para as aplicações existentes quanto materiais novos que permitirão novas aplicações são esperados. Certamente, uma das áreas com maior potencial de benefício será a de biomateriais, onde a estrutura fibrilar na escala submicrométrica, a biocompatibilidade que advém do uso de polímeros de origem natural e a possibilidade de carregamento com substâncias bioativas, implicam em um conjunto de propriedades até então inédito em qualquer material de engenharia, abrindo um novo universo de possibilidades de engenharia biomédica.

$\mathrm{O}$ uso de nanoestruturas fibrilares também apresenta interessantes possibilidades na aplicação em sensores. Os principais benefícios são a altíssima área superficial, principalmente quando fibras porosas são utilizadas. As nanofibras podem ser utilizadas tanto como suporte de alta área, por exemplo, para enzimas, quanto como material transdutor em si, por exemplo, monitorando variações de condutividade ou constante dielétrica que ocorrem no material quando da sorção de determinado analito. Outra possibilidade é a modulação das propriedades óticas das fibras quando em contato com a substância de interesse, detectável por colorimetria, fluorimetria, refratometria, entre outras possibilidades.

Catálise é uma área onde nanofibras são estruturas com potencial imenso para trazer benefícios industriais, dada a necessidade intrínseca de alta área superficial. Resultados obtidos até o momento são encorajadores, porém da criação de um novo material até a aplicação em processos industriais, longo tempo de desenvolvimento é tipicamente necessário. Espera-se que daqui a alguns anos os primeiros relatos de aplicação comercial de catalisadores imobilizados em nanofibras obtidas por eletrofiação estejam sendo divulgados.

O impacto desta tecnologia deve ocorrer de maneira generalizada em muitos setores, porém mais sensivelmente em segmentos que façam uso, ou tenham contato, com materiais de origem biológica. Para estes, a natureza orgânica dos polímeros e dimensão submicrométrica das fibras cria um microambiente compatível e de interações similares, permitindo que o casamento das propriedades seja feito de forma mais suave e natural. Para o Brasil, o setor do agronegócio é talvez o mais importante neste cenário, seguido dos setores de alimentos e bebidas e biomédico. As aplicações potenciais são inúmeras, seja para fibras poliméricas ou derivadas destas, para nanocompósitos ou blendas, seja revestindo superfícies ou em dispositivos, enfim, toda uma gama de possibilidades ainda não completamente imaginada.

Para finalizar, é importante ressaltar que a caracterização destes materiais se vale das técnicas já tradicionalmente utilizadas para caracterização microestrutural de materiais, não sendo necessária nenhuma infraestrutura tecnológica adicional. Este ponto é muito positivo, pois permite que praticamente qualquer laboratório equipado com instrumentação para análise microestrutural de polímeros possa também atuar no desenvolvimento, caracterização e aplicação de nanofibras, criando assim uma grande base para o desenvolvimento desta plataforma tecnológica no país.

\section{Referências Bibliográficas}

1. Costa, R. G. F.; Oliveira, J. E.; Paula, G. F.; Picciani, P. H. S.; Medeiros, E. S.; Ribeiro, C.\& Mattoso, L. H. C. - Polímeros (2012). In press.

2. Li, D.; Wang, Y. L. \& Xia, Y. N. - Nano Lett., 3, p.1167 (2003). http:// dx.doi.org/10.1021/n10344256

3. Li, D. \& Xia, Y. - Nano Lett., 3, p.555 (2003). http://dx.doi.org/10.1021/ n1034039o

4. Tuttle, R. W.; Chowdury, A.; Bender, E. T.; Ramsier, R. D.; Rapp, J. L. \& Espe, M.P. - Appl. Surf. Sci., 254, p.4925 (2008). http://dx.doi. org/10.1016/j.apsusc.2007.12.068

5. Azada, A. M. - Mat. Sci. Eng. A-Struct., 435-436, p.468 (2006).

6. Barnes, W. L.; Dereux, A. \& Ebbesen, T. W. - Nature, 424, p.824 (2003). http://dx.doi.org/10.1038/nature01937

7. Kelly, K. L.; Coronado, E.; Zhao, L. L. \& Schatz, G. C. - J. Phys. Chem. B, 107, p.668 (2003). http://dx.doi.org/10.1021/jp026731y

8. Sun, Y.G. \& Xia, Y. - Science, 298, p.2176 (2002). http://dx.doi. org/10.1126/science.1077229

9. Bognitzki, M.; Becker, M.; Graeser, M.; Massa, W.; Wendorff, J.H.; Schaper, A.; Weber, D.; Beyer, A.; Gölzhäuser, A. \& Greiner, A. - Adv. Mater., 18, p.2384 (2006). http://dx.doi.org/10.1002/adma.200600103

10. Pol, V. G.; Koren, E. \& Zaban, A. - Chem. Mater., 20, p.3055 (2008). http://dx.doi.org/10.1021/cm7036958 
11. Wang, Z. X. \& Liu, L. - Mater. Lett., 63, p.917 (2009). http://dx.doi org/10.1016/j.matlet.2009.01.051

12. Graeser, M.; Bognitzki, M.; Massa, W.; Pietzonka, C.; Greiner, A. \& Wendorff, J.H. - Adv. Mater., 19, p.4244 (2007). http://dx.doi. org/10.1002/adma.200700849

13. Wu, H.; Zhang, R.; Liu, X.; Lin, D. \& Pan, W. - Chem. Mater., 19, p.3506 (2007). http://dx.doi.org/10.1021/cm070280i

14. Shao, C.; Kim, H-Y.; Gong, J.; Ding, B.; Lee, D-R. \& Park, S-J. - Mater. Lett., 57, p.1579 (2003). http://dx.doi.org/10.1016/S0167$577 \mathrm{X}(02) 01036-4$

15. Medeiros, E. S.; Mattoso, L. H.C.; Ito, E. N.; Gregorski, K. S.; Robertson, G. H.; Offeman, R.D.; Wood, D. F.; Orts, W. J.; Imam, S. H. - J. Biobased Mater. Bio., 2, p.231 (2008). http://dx.doi.org/10.1166/ jbmb.2008.411

16. Ko, F.; Gogotsi, Y.; Ali, A.; Naguib, N.; Ye, H.; Yang, G. L.; Li, C.; Willis, P. - Adv. Mater., 15, p.1161 (2003). http://dx.doi.org/10.1002/ adma.200304955

17. Sen, R.; Zhao, B.; Perea, D.; Itkis, M. E.; Hu, H.; Love, J.; Bekyarova, E. \& Haddon, R. C. - Nano Lett., 4, p.459 (2004). http://dx.doi. org/10.1021/n1035135s

18. Son, W. K.; Youk, J. H. \& Park, W. H. - Carbohydr. Polym., 65, p.430 (2006). http://dx.doi.org/10.1016/j.carbpol.2006.01.037

19. Costa, R. G. F.; Ribeiro, C. \& Mattoso, L. H. C. - Sci. Adv. Mater., 2, p.157 (2010). http://dx.doi.org/10.1166/sam.2010.1065

20. Li, L.; Bellan, L. M.; Craighead, H. G. \& Frey, M. W. - Polymer, 47, p.6208 (2006). http://dx.doi.org/10.1016/j.polymer.2006.06.049

21. Lee, H. W.; Karim, M. R.; Ji, H. M.; Choi, J. H.; Ghim, H. D.; Park, S. M.; Oh, W. \& Yeum, J. H. - Appl. Polym. Sci., 113, p.1860 (2009). http://dx.doi.org/10.1002/app.30165

22. Utracki, L. A. - "Polymer Blends Handbook", Kluwer Academic Publisher, Dordrecht (2002).

23. Picciani, P. H. S.; Soares, B. G.; Medeiros, E. S.; Souza Junior, F. G.; Wood, D. F.; Orts, W. J. \& Mattoso, L. H. C. - Macromol. Theor Simul., 18, p.528 (2009). http://dx.doi.org/10.1002/mats.200900053

24. Picciani, P. H. S.; Medeiros, E. S.; Pan, Z.; Wood, D. F.; Orts, W. J.; Mattoso, L. H. C. \& Soares, B. G. - Macromol. Mater. Eng., 7, p. 618 (2010). http://dx.doi.org/10.1002/mame.201000019

25. Vinckier, I.; Moldenaers, P. \& Mewis, J. - J. Rheol., 40, p.613 (1996). http://dx.doi.org/10.1122/1.550800

26. Hong, C. K.; Yang, K. S.; Oh, S. H.; Ahn, J-H.; Cho, B-H. \& Nah, C.; - Polym. Int., 57, p.1357 (2008). http://dx.doi.org/10.1002/pi.2481

27. Wei, M.; Kang, B.; Sung, C. \& Mead, J. - Macromol. Mater. Eng., 291, p.1307 (2006). http://dx.doi.org/10.1002/mame.200600284

28. Chen, H.; Snyder, J. D. \& Elabd, Y. A. - Macromolecules, 41, p.128 (2008). http://dx.doi.org/10.1021/ma070893g

29. Choi, J. S.; Lee, S. J.; Christ, G. J.; Atala, A. \& Yoo, J. J. - Biomaterials, 29 , p.2899 (2008). http://dx.doi.org/10.1016/j.biomaterials.2008.03.031

30. Desai, K.; Kit, K.; Li, J.; Davidson, P. M.; Zivanovic, S. \& Meyer, H. - Polymer, 50, p.3661 (2009). http://dx.doi.org/10.1016/j. polymer.2009.05.058

31. Yoshimoto, H.; Shina, Y. M.; Teraia, H. \& Vacanti, J. P. Biomaterials, 24, p.2077 (2003). http://dx.doi.org/10.1016/S01429612(02)00635-X

32. Venugopal, J. \& Ramakrishna, S. - Appl. Biochem. Biotech., 125, p.147 (2005). http://dx.doi.org/10.1385/ABAB:125:3:147

33. Dai, N. T.; Williamson, M. R.; Khammo, N.; Adams, E. F. \& Coombes, A. G. A. - Biomaterials, 25, p. 4263 (2004). http://dx.doi.org/10.1016/j. biomaterials.2003.11.022

34. Sawicka, K.; Gouma, P. \& Simon, S. - Sensors Actuat. B-Chem., 108, p.585 (2005).
35. Sill, T.J. \& von Recum, H.A. - Biomaterials, 29, p.1989 (2008). http:// dx.doi.org/10.1016/j.biomaterials.2008.01.011

36. Pham, Q.P.; Sharma, U. \& Mikos, A.G. - Tissue Eng., 12, p.1197 (2006). http://dx.doi.org/10.1089/ten.2006.12.1197

37. Khil, M. S.; Cha, D.; Kim, H-Y.; Kim, I-S. \& Bhattarai, N. - J. Biomed. Mater. Res. B, 67B, p.675 (2003). http://dx.doi.org/10.1002/ jbm.b. 10058

38. Choi, J. S.; Leong, K. W. \& Yoo, H. S. - Biomaterials, 29, p.587 (2008). http://dx.doi.org/10.1016/j.biomaterials.2007.10.012

39. Nisbet, D. R.; Forsythe, J. S.; Shen, W.; Finkelstein, D. I. \& Horne, M. K. - J. Biomater. Appl., 24, p.7 (2009). http://dx.doi. org/10.1177/0885328208099086

40. Kim, K.; Luu, Y. K.; Chang, C.; Fang, D.; Hsiao, B. S.; Chu, B. \& Hadjiargyrou, M. - J. Control. Release, 98, p.47 (2004). http://dx.doi. org/10.1016/j.jconrel.2004.04.009

41. Jia, H. F.; Zhu, G.; Vugrinovich, B.; Kataphinan, W.; Reneker, D. H. \& Wang, P.-Biotechnol. Progr., 18, p.1027 (2002). http://dx.doi. org/10.1021/bp020042m

42. Ren, G. L.; Xu, X.; Liu, Q.; Cheng, J.; Yuan, X.; Wu, L. \& Wan, Y. React. Funct. Polym., 66, p.1559 (2006). http://dx.doi.org/10.1016/j. reactfunctpolym.2006.05.005

43. Luu, Y. K.; Kim, K.; Hsiao, B. S.; Chu, B. \& Hadjiargyrou, M. - J. Control. Release, 89, p.341 (2003). http://dx.doi.org/10.1016/S01683659(03)00097-X

44. Wang, Z. G.; Wan, L-S.; Liu, Z-M.; Huang, X-J. \& Xu, Z-K. - J. Mol. Catal. B-Enzym., 56, p.189 (2009). http://dx.doi.org/10.1016/j. molcatb.2008.05.005

45. Koide, M.; Osaki, K.; Konishi, J.; Oyamada, K.; Katakura, T.; Takahashi, A. \& Yoshizato, K. - J. Biomed. Mater. Res., 27, p.79 (1993). http://dx.doi.org/10.1002/jbm.820270111

46. Ono, K.; Saito, Y.; Yura, H.; Ishikawa, K.; Kurita, A.; Akaike, T. \& Ishihara, M. - J. Biomed. Mater. Res., 49, p.289 (2000).http:// dx.doi.org/10.1002/(SICI)1097-4636(200002)49:2\%3C289::AIDJBM18\%3E3.0.CO;2-M

47. Wang, Y. Z.; Kim, H-J.; Vunjak-Novakovic, G. \& Kaplan, D. L. - Biomaterials, 27, p.6064 (2006). http://dx.doi.org/10.1016/j. biomaterials.2006.07.008

48. Kim, K.; Yu, M.; Zong, X.; Chiu, J.; Fang, D.; Seo, Y-S.; Hsiao, B. S.; Chu, B. \& Hadjiargyrou, M. - Biomaterials, 24, p.4977 (2003). http:// dx.doi.org/10.1016/S0142-9612(03)00407-1

49. Reneker, D. H. \& Yarin, A. L. - Polymer, 49, p.2387 (2008). http:// dx.doi.org/10.1016/j.polymer.2008.02.002

50. Xu, C. Y; Inai, R.; Kotaki, M \& Ramakrishna, S. - Biomaterials., 5, p.887 (2004).

51. Sundaray, B.; Subramanian, V.; Natarajan, T. S.; Xiang, R-Z.; Chang, C-C. \& Fann, W-S. - Appl. Phys. Lett., 84, p.1222 (2004). http://dx.doi org/10.1063/1.1647685

52. Yan, H.; Liu, L. Q. \& Zhang, Z. - Appl. Phys. Lett., 95, p.143114 (2009). http://dx.doi.org/10.1063/1.3242378

53. Zong, X. H.; Ran, S.; Fang, D.; Hsiao, B. S. \& Chu, B. - Polymer, 44, p.4959 (2003). http://dx.doi.org/10.1016/S0032-3861(03)00464-6

54. Lim, C. T.; Tan, E. P. S. \& Ng, S. Y. - Appl. Phys. Lett., 92, p.141908 (2008). http://dx.doi.org/10.1063/1.2857478

55. Wang, X.; Drew, C.; Lee, S-H.; Senecal, K. J.; Kumar, J. \& Samuelson, L. A. - Nano Lett., 2, p.1273 (2002). http://dx.doi.org/10.1021/ $\mathrm{nl020216u}$

56. Liu, H.; Kameoka, J.; Czaplewski, D. A. \& Craighead, H. G. - Nano Lett., 4, p.671 (2004). http://dx.doi.org/10.1021/n1049826f

57. Wang, X.; Kim, Y-G.; Drew, C.; Ku, B-C.; Kumar, J.; Samuelson, L. A. - Nano Lett., 4, p.331 (2004). http://dx.doi.org/10.1021/n1034885z 
58. Luoh, R. \& Hahn, H. T. - Compos. Sci. Technol., 66, p.2436 (2006). http://dx.doi.org/10.1016/j.compscitech.2006.03.012

59. Manesh, K. M.; Gopalan, A. I.; Lee, K-P.; Santhosh, P.; Song, K-D. \& Lee, D-D. - IEEE Trans. Nanotechnol., 6, p.513 (2007). http://dx.doi. org/10.1109/TNANO.2007.903918

60. Tao, S.; Li, G. \& Yin, J. - J. Mater. Chem., 17, p.2730 (2007). http:// dx.doi.org/10.1039/b618122h

61. Haynes, A. S. \& Gouma, P. I. - IEEE Sensors J., 8, p.701 (2008).

62. Yoshimatsu, K.; Ye, L.; Stenlund, P. \& Chronakis, S. - J. Chem. Soc., Chem. Commun., p.2022 (2008).

63. Kuo, C. C.; Wang, C. T. \& Chen, W. C. - Macromol. Mater. Eng., 293, p.999, (2008). http://dx.doi.org/10.1002/mame.200800224

64. Tiwari, M. K.; Yarin, A. L. \& Megaridis, C. M. - J. Appl. Phys., 106, p.44305 (2008).

65. Rathfon, J. M.; AL-Badri, Z. M.; Shunmugam, R.; Berry, S. M.; Pabba, S.; Keynton, R. S.; Cohn, R. W. \& Tew, G. N. - Adv. Funct. Mater., 19, p.689 (2009). http://dx.doi.org/10.1002/adfm.200800947

66. Yoon, J.; Jung, Y. S. \& Kim, J. M. - Adv. Funct. Mater., 19, p.209 (2009). http://dx.doi.org/10.1002/adfm.200800963

67. Picciani, P. H. S.; Medeiros, E. S.; Pan, Z.; Orts, W. J.; Mattoso, L. H. C. \& Soares, B. G. - J. Appl. Polym. Sci., 112, p.744 (2009). http:// dx.doi.org/10.1002/app.29447

68. Costa, L. M. M.; Bretas, R. E. S. \& Gregorio Filho, R. - Polímeros, 19, p.183 (2009).

69. Demir, M.M.; Gulgun, M.A.; Menceloglu, Y. Z.; Erman, B.; Abramchuck, S. S.; Makhaeva, E. E.; Khokhlov, A. R.; Matveeva, V. G. \& Sulman, M. G. - Macromolecules, 37, p.1787 (2004).

70. Chen, J.; Li, Z.; Chao, D.; Zhang, W. \& Wang, C. - Mater. Lett., 62, p.692 (2008). http://dx.doi.org/10.1016/j.matlet.2007.06.047

71. Zhou, Z.; Feng, Y.; Xu, W.; Ren, F. \& Ma, H. - J. Appl. Polym. Sci., 113, p.1264 (2009). http://dx.doi.org/10.1002/app.30139

72. Costa, R. G. F.; Ribeiro, C. \& Mattoso, L. H. C. - J. Nanosci. Nanotechno., 10, p.5144 (2010). http://dx.doi.org/10.1166/ jnn.2010.2405

73. Wu, L.; Yuan, X. \& Sheng, J. - J. Membr. Sci., 250, p.167 (2005). http:// dx.doi.org/10.1016/j.memsci.2004.10.024

74. Kim, B. C.; Nair, S.; Kim, J.; Kwak, J. H.; Grate, J. W.; Kim, S. H. \& Gu, M. B. - Nanotechnology, 16, p.S382 (2005). http://dx.doi. org/10.1088/0957-4484/16/7/011

75. Lee, S. \& Obendorf, S. K. - Text. Res. J., 77, p.696 (2007). http:// dx.doi.org/10.1177/0040517507080284

76. Veleirinho, B. \& Lopes-da-Silva, F. A. - Process Biochem., 44, p. 353 (2009). http://dx.doi.org/10.1016/j.procbio.2008.11.008

77. Ohgo, K.; Zhao, C.; Kobayashi, M. \& Asakura, T. - Polymer, 44, p.841 (2003). http://dx.doi.org/10.1016/S0032-3861(02)00819-4

78. Torres-Giner, S.; Gimenez, E. \& Lagarona, J. M. - Food Hydrocolloid., 22, p.601 (2008). http://dx.doi.org/10.1016/j. foodhyd.2007.02.005
79. Vega-Lugo, A. C. \& Lim, L. T. - Food Res. Int., 42, p.933 (2009). http://dx.doi.org/10.1016/j.foodres.2009.05.005

80. Taepaiboon, P.; Rungsardthong, U. \& Supaphol, P. - Eur. J. Pharm. Biopharm., 67, p.387 (2007). http://dx.doi.org/10.1016/j. ejpb.2007.03.018

81. Ramakhishna, S.; Fujihara, K.; Teo, W-E.; Lim, T-C. \& Ma, Z. - "An Introduction to Electrospinning and Nanofibers", World Scientific Publishing, Singapore (2005). http://dx.doi. org/10.1142/9789812567611

82. Lee, K. Y.; Jeong, L.; Kang, Y. O.; Lee, S. J. \& Park, W. H. - Adv. Drug Deliver. Rev., 61, p.1020 (2009). http://dx.doi.org/10.1016/j. addr.2009.07.006

83. Medeiros, E. S.; Mattoso, L. H. C.; Offeman, R. D.; Wood, D. F.; Orts, W. J. - Can. J. Chemistry, 86, p.590 (2008). http://dx.doi.org/10.1139/ v08-029

84. Yang, Q. B.; Li, D. M.; Hong, Y. L.; Li, Z. Y.; Wang, C.; Qiu, S. L. \& Wei, Y. - Synthetic Met., 137, p.973 (2003). http://dx.doi.org/10.1016/ S0379-6779(02)00963-3

85. Jose, M. V.; Steinert, B. W.; Thomas, V.; Dean, D. R.; Abdalla, M. A.; Price, G. \& Janowski, G. M. - Polymer, 48, p.1096 (2007). http:// dx.doi.org/10.1016/j.polymer.2006.12.023

86. Butt, H. J.; Cappella, B. \& Kappl, M. - Surf. Sci. Rep., 59, p.1 (2005). http://dx.doi.org/10.1016/j.surfrep.2005.08.003

87. Garcia, R. \& Perez, R. - Surf. Sci. Rep., 47, p.197 (2002).

88. Lee, S. W. \& Belcher, A. M. - Nano Lett., 4, p.387 (2004). http://dx.doi. org/10.1021/n1034911t

89. Wu, Y. Q.; Johannes, M. S. \& and Clark, R. L. - Mater. Lett., 62, p.699 (2008). http://dx.doi.org/10.1016/j.matlet.2007.06.052

90. Dersch, R.; Liu, T.; Schaper, A. K.; Greiner, A. \& Wendorff, J. H. - J. Polym. Sci. Pol. Chem, 41, p.545 (2003). http://dx.doi.org/10.1002/ pola.10609

91. Esrafilzadeh, D.; Jalili, R. \& Morshed, M. - J. Appl. Polym. Sci., 110, p.3014 (2008). http://dx.doi.org/10.1002/app.27593

92. Chen, C. Z.; Wang, L. G. \& Huang, Y. - Sol. Energ. Mat. Sol. C., 92, p.1382 (2008). http://dx.doi.org/10.1016/j.solmat.2008.05.013

93. Zhang, W.X.; Wang, Y.Z. \& Sun, C.F. - J. Polym. Res., 14, p.467 (2007). http://dx.doi.org/10.1007/s10965-007-9130-X

94. Zoccola, M.; Aluigi, A.; Vineis, C.; Tonin, C.; Ferrero, F. \& Piacentino, M. G. - Biomacromolecules, 9, p.2819 (2008). http://dx.doi. org $/ 10.1021 / \mathrm{bm} 800579 \mathrm{a}$

95. Dong, F.; Li, Z.; Huang, H.; Yang, F.; Zheng, W. \& Wang, C. - Mater. Lett., 61, p.2556 (2007). http://dx.doi.org/10.1016/j.matlet.2006.09.058

96. Duan, B.; Dong, C.; Yuan, X. \& Yao, K. - J. Biomater. Sci., Polym. Ed., 15, p.797 (2004). http://dx.doi.org/10.1163/156856204774196171

Enviado: 09/12/10

Reenviado: 23/06/11

Aceito: $27 / 07 / 11$ 